\title{
Assumed similarity in personality within intimate relationships
}

Liu, Jie; Ludeke, Steven; Zettler, Ingo

Published in:

Personal Relationships

DOI:

10.1111/pere. 12246

Publication date:

2018

Document version

Peer reviewed version

Citation for published version (APA):

Liu, J., Ludeke, S., \& Zettler, I. (2018). Assumed similarity in personality within intimate relationships. Personal Relationships, 25(3), 316-329. https://doi.org/10.1111/pere.12246 
This work is scheduled to appear in

Personal Relationships

(c) 2018 International Association for Relationship Research / John Wiley \& Sons, Inc.

This manuscript may not exactly replicate the final version published in the journal. It is not the copy of record.

Reference:

Liu, J., Ludeke, S., \& Zettler, I. (2018). Assumed similarity in personality within intimate relationships. Personal Relationships, 25, 316-329. https://doi.org/10.1111/pere.12246 
Assumed Similarity in Personality within Intimate Relationships

Jie Liu

University of Copenhagen

Steven Ludeke

University of Southern Denmark

Ingo Zettler

University of Copenhagen 
ASSUMED PERSONALITY SIMILARITY IN COUPLES

\begin{abstract}
Assumed similarity is the tendency to assume that another person is similar to oneself. We examined assumed similarity in intimate relationships regarding the HEXACO personality traits in Denmark $(N=93)$ and China $(N=236)$. Specifically, we hypothesized that people assume higher similarity with their intimate partners in Honesty-Humility and Openness to Experience compared to the other four HEXACO traits (Emotionality, Extraversion, Agreeableness, and Conscientiousness). Results from both studies indicate that assumed similarity was higher in Honesty-Humility compared to Emotionality, Extraversion, Agreeableness and Conscientiousness. However, assumed similarity in Openness was higher compared to Emotionality and Conscientiousness only. Supplementary analyses indicate no cultural differences between Denmark and China in assumed similarity in Honest-Humility and Openness.
\end{abstract}

Keywords: assumed similarity; intimate relationships; HEXACO; Honesty-Humility; Openness to Experience. 
ASSUMED PERSONALITY SIMILARITY IN COUPLES

A large body of research has suggested that people tend to think in ways that are consistent with their desires, termed motivated cognition (e.g., Kunda, 1990; Lemay \& Clark, 2015). Concerning intimate relationships, the phenomenon of motivated cognition suggests that people who want to maintain their relationships see their partner in their desired ways. For example, Lemay and Neal (2014) found that people who wanted to bond with their partner tended to perceive their partner as more responsive when discussing personal problems_even after controlling for the partner's self-report of behavior as well as ratings of the partner's behavior by objective raters. In a similar vein, it has been found that people who value their intimate relationship are more likely to perceive their partner as having more desirable characteristics (e.g., intelligent, physically attractive, skilled, trustworthy; Murray, Holmes, Dolderman, \& Griffin, 2000) or are less attentive to attractive relationship alternatives (e.g., Johnson \& Rusbult, 1989; Maner, Gailliot, \& Miller, 2009).

Importantly, motivated cognition does not suggest in general that people ascribe very low or high levels of a characteristic to their partners. In fact, motivated cognition rather suggests that people ascribe specific levels of a characteristic to their partners. One specific level could be to describe one's partner as similar to oneself because such tendency has been found to enhance interpersonal connection, relationship satisfaction, and understanding between partners (Byrne, 1971; Montoya, Horton, \& Kirchner, 2008; Murray, Holmes, Bellavia, Griffin, \& Dolderman, 2002). The tendency to perceive another person (such as one's partner) as similar to oneself is termed assumed similarity, typically estimated by the correlation between one's self-report and one's observer report of another person (e.g., Kenny \& Acitelli, 2001; Kenny \& West, 2010; Paunonen \& Hong, 2013; Srivastava, Guglielmo, \& Beer, 2010). 
ASSUMED PERSONALITY SIMILARITY IN COUPLES

Research on assumed similarity in intimate relationships has largely focused on basic personality traits, and supported the existence of assumed similarity. For example, in a study by Watson, Hubbard, and Wiese (2000), the assumed similarity in dating/married couples ranged from $r=.11$ to .50 across the Big Five traits (Openness to Experience, Conscientiousness, Extraversion, Agreeableness, and Neuroticism). Interestingly, the actual similarity — typically estimated by the correlation between one's self-report and the self-report of another person (in this case, one's partner) on the same characteristic — ranged from $r=.06$ to .36 only. In a similar vein, McCrae et al. (2008) reported relatively consistent higher assumed similarity, compared to actual similarity, in both domains (traits) and facets of the Big Five between spouses across the Czech Republic, Russia, and the US. In brief, studies have indicated that couples show assumed similarity regarding personality traits, and that the extent of assumed similarity is typically higher than the extent of actual similarity in intimate relationships.

Research has not only indicated that assumed similarity concerning personality traits exists, but also that assumed similarity might be different in magnitude for different traits. Specifically, it has been found that people show higher assumed similarity in some traits than in others. For example, in a sample comprising different forms of dyads (e.g., friendship dyads, dating couples, married couples), Watson et al. (2000) reported an average assumed similarity of $r=.31$ for Openness to Experience, while the average assumed similarity for the other Big Five traits was comparatively weak $(.13 \leq r \leq .21)$. Similarly, McCrae et al. (2008) reported that spouses from the three investigated countries (i.e., the Czech Republic, Russia, and the US) showed assumed similarity in Openness to Experience between $r=.21$ and .22 , whereas there was no such consistent pattern concerning the other traits $(-.06 \leq r \leq .29)$ except for Agreeableness $(.18 \leq r \leq .35)$. 
ASSUMED PERSONALITY SIMILARITY IN COUPLES

Recently, studies have started to examine assumed similarity regarding personality traits using the HEXACO Model of Personality (Ashton \& Lee, 2007). The HEXACO model comprises six traits: Honesty-Humility, Emotionality, Extraversion, Agreeableness, Conscientiousness and Openness to Experience (in the following simply: Openness). Generally, Extraversion, Conscientiousness and Openness virtually mirror their Big Five counterparts. Emotionality and Agreeableness represent alternative rotations of Big Five Neuroticism and Agreeableness, resulting in slight differences between Emotionality vs Neuroticism and HEXACO-Agreeableness vs Big Five-Agreeableness (for more details, please see Ashton \& Lee, 2007; Ashton, Lee, \& De Vries, 2014). Honesty-Humility represents the most striking difference between the HEXACO model and the Big Five, as the latter does not include a corresponding counterpart. Honesty-Humility comprises characteristics such as being honest, loyal, and sincere versus being boastful, hypocritical, and pretentious (Lee \& Ashton, 2004, 2006).

Using the HEXACO model to investigate assumed similarity, Lee et al. (2009) found in two Canadian samples of well-acquainted dyads (e.g., friends, relatives, and romantic partners) that assumed similarity in Honesty-Humility $(r=.44$ and $r=.46)$ and Openness $(r=.35$ and $r=$ .30) was higher compared to that of the remaining four HEXACO traits $(-.07 \leq r \leq .12)$. A similar pattern was observed in a sample of co-worker dyads from the US, in which assumed similarity was highest in Honesty-Humility $(r=.61)$ and Openness $(r=.48$; for the other traits: $.25 \leq r \leq .46$; Cohen, Panter, Turan, Morse, $\&$ Kim, 2013). In another sample combining dyads of co-workers, relatives, romantic partners, and other well-acquainted individuals in the US, Cohen et al. (2013) again found higher assumed similarity in Honesty-Humility $(r=.48)$; however, in this sample assumed similarity was also found in Agreeableness $(r=.15)$ and Conscientiousness $(r=.10)$, but not in Openness $(r=.07)$. Noticeably, the consistent finding of 
ASSUMED PERSONALITY SIMILARITY IN COUPLES

high assumed similarity in Honesty-Humility was also indirectly supported by McCrae et al. (2008), reporting assumed similarity in spouses for the Agreeableness facets

Straightforwardness(.29 $\leq r \leq .38)$ and Modesty(.19 $\leq r \leq .45)$, which conceptually and empirically overlap with Honesty-Humility (Ashton \& Lee, 2005; Ashton et al., 2014).

Overall, previous studies — using different groups of dyads — provide a relatively clear picture. Concerning the Big Five, assumed similarity occurs most for Openness and (facets of) Agreeableness. Concerning the HEXACO model, assumed similarity occurs most for HonestyHumility and, albeit weaker and/or less consistently, for Openness. Concerning the other traits from the two personality models, assumed similarity tends to be less consistent and weaker, or even non-existent.

Although previous studies investigating assumed similarity with regard to the HEXACO traits provide a relatively clear picture, it has not been tested yet whether higher assumed similarity in Honesty-Humility and — albeit weaker — in Openness also occurs when considering intimate relationships exclusively. Indeed, some arguments suggest that higher assumed similarity in Honesty-Humility and Openness still holds when focusing exclusively on intimate relationships, while some arguments do not. Specifically, on the one hand, the tendency to assume higher similarity in Honesty-Humility and Openness should be likely to occur in the context of intimate relationships as well given the ubiquity of motivated cognition in intimate relationships and assumed similarity as one specific type of motivated cognition. Lee et al. (2009) have provided some initial support for this idea by comparing assumed similarity in Honesty-Humility and Openness between friends and non-friend acquaintances, and found that people were more likely to assume higher similarity in these two personality traits with their close acquaintances than with more distant ones. Lee et al. (2009) concluded that people tend to 
ASSUMED PERSONALITY SIMILARITY IN COUPLES

overestimate their similarity to persons with whom they have a close relationship. By

implication, people may exhibit a strong tendency to assume higher similarity in Honesty-

Humility and Openness when it comes to their intimate partners given the great intimacy shared by intimate partners (Bradbury \& Karney, 2010).

On the other hand, however, the great intimacy that people share with their intimate partners might also facilitate accurate personality judgements, because partners not only have more opportunities to observe each other, but are also more willing to disclose most of their inner feelings to each other (Kenny \& Acitelli, 2001). Consequently, the overall assumed similarity in personality, including that in Honesty-Humility and Openness, might become lower in the context of intimate relationships, because people might describe their partner more accurately and thus leaving less room for the tendency to assume non-existent or to exaggerate existent similarity. As an indirect support for this line of reasoning, Cohen et al. (2013) reported lower assumed similarity in the HEXACO traits for the sample consisting of around $32 \%$ intimate partners $(-.02 \leq r \leq .48)$ compared to the sample only including co-workers $(.25 \leq r \leq$ .61). Consequently, it seems questionable whether assumed similarity in Honesty-Humility and Openness is still existent and stronger as compared to the other traits in the context of intimate relationships considering the two opposing forces, motivated cognition and perception accuracy.

To sum up, previous theorizing and empirical findings have suggested that people tend to assume higher similarity in Honesty-Humility and Openness, compared to the other basic personality traits. However, previous studies have, at least, two limitations which we address in the current investigation. First, previous studies using the HEXACO model—which seems superior to the Big Five when investigating assumed similarity due to its inclusion of HonestyHumility — relied on a mixed group of dyads (e.g., friends, partners). Thus, it is unclear whether 
ASSUMED PERSONALITY SIMILARITY IN COUPLES

higher assumed similarity in Honesty-Humility and Openness can also be found when focusing on intimate relationships exclusively given the potential existence of two counteractive effects (motivated cognition and perception accuracy). Second, no study has statistically compared assumed similarity in Honesty-Humility and Openness with assumed similarity in the other personality traits. Thus, it is unclear whether there are significant differences in assumed similarity between different personality traits. In our investigation, we addressed these two limitations by only recruiting participants being in intimate relationships and by statistically comparing assumed similarity between different personality traits. Despite the possibility of higher perception accuracy in intimate dyads, most of the prior literature has suggested higher assumed similarity in Honesty-Humility and Openness as compared to the other traits, including the one focusing on close others (Lee et al., 2009). Thus, based on these findings and corresponding arguments, we expect to find higher assumed similarity in intimate dyads in Honesty-Humility (Hypothesis 1) and Openness (Hypothesis 2) as compared to the other four HEXACO traits in two samples from Denmark (including one partner of an intimate dyad) and China (including both partners of an intimate dyad).

\section{Study 1: Danish Study}

\section{Participants and Procedure}

Study 1 was conducted online. Students from two introductory lectures in Personality Psychology in two Danish universities were invited to participate in the current study voluntarily, anonymously, and without payment. From the 275 participants $^{i}$ who started the study, we included 151 participants who indicated that they were currently involved in an intimate relationship. Finally, 93 participants who passed two attention checking items interspersed in the assessment $^{\mathrm{ii}}$ were included in the current study, and their age ranged from 19 to $60(M=29.6$, 
ASSUMED PERSONALITY SIMILARITY IN COUPLES

$S D=10.2$ ) years. Participants first completed self- and then current partner ratings concerning the HEXACO traits, with two short questionnaires (irrelevant to the current study) as a filler task in between, so that participants would not immediately see virtually identical questionnaires, one concerning themselves and one concerning their partner, successively. All the materials and related analyses are available in the OSF framework (https://osf.io/9nqau/).

\section{Measures}

Participants' personality was assessed via the 60-item version of the HEXACO Personality Inventory-Revised (HEXACO-60, Ashton \& Lee, 2009), using a response scale from 1 (strongly disagree) to 5 (strongly agree). We adapted the observer report version of the HEXACO-60 to assess participants' observer rating of their partners' personality. Sample items are "I would be quite bored by a visit to an art gallery" (Openness, self-report) and "My partner would be quite bored by a visit to an art gallery" (Openness, observer report). All questionnaires were in English because both Personality Psychology lectures were held in English. Further, note that Danes generally show a high level in English proficiency (Preisler, 2003). Correspondingly, above $94 \%$ of the participants reported their English level as very good or native, while the final $6 \%$ still as satisfactory. Cronbach's alphas of the self- and partner report ranged from .75 to .87.

\section{Methods}

We used the double entry intraclass correlation ${ }^{\mathrm{iii}}$ (ICC; Furr, 2010) to index similarity between self- and partner ratings. Compared to the Pearson correlation, which only accounts for pattern similarity of two variables, the ICC also takes similarity in the mean and variability of two variables into consideration. Thus, the ICC is a more comprehensive way of calculating similarity and assumed similarity within dyads. 
ASSUMED PERSONALITY SIMILARITY IN COUPLES

For comparing the ICCs of Honesty-Humility and Openness with the ICCs of the remaining HEXACO traits, we used the cocor package in R (Diedenhofen \& Musch, 2015) and adopted the method of comparing two non-overlapping correlations based on dependent groups (i.e., the same group), because the ICCs of the HEXACO traits in the current study do not share common variables, but are from the same participant pool.

\section{Results and Discussion}

The lower left of Table 1 shows the correlations between self- and partner ratings for all HEXACO traits. We compared the ICCs of Honesty-Humility and Openness with these of the other four traits. Here, we report specific $p$-values for all tests. Because readers may disagree about whether the nature of the present study may or may not merit raising the threshold for statistical significance (e.g., from $p<.05$ to $p<.01$ ) based on concerns about multiple testing, each reader thus has the information needed to evaluate our results according to her/his view. Because very few of the results presented below have $p$-values falling between .05 and .01 , the conclusions are generally unaffected by such considerations. The ICC of Honesty-Humility $\left(r_{I C C}\right.$ $=.50)$ was significantly higher than the ICCs of Emotionality $(z=6.55, p<.001, \mathrm{CI}[.53,1.01]$, Cohen's $q=.848)$, Extraversion $(z=2.60, p=.009$, CI $[.07, .58]$, Cohen's $q=.378)$, Agreeableness $(z=4.32, p<.001$, CI [.27, .73], Cohen's $q=.559)$, and Conscientiousness $(z=$ $6.16, p<.001$, CI $[.49, .97]$, Cohen's $q=.805)$. However, the ICC of Openness $\left(r_{I C C}=.17\right)$ was only significantly higher than the ICCs of Emotionality $(z=3.36, p<.001, \mathrm{CI}[.18, .72]$, Cohen's $q=.470)$ and Conscientiousness $(z=3.00, p=.003$, CI $[.13, .68]$, Cohen's $q=.427)$, but was not higher than the ICCs of Extraversion ( $z=.00, p=1.00$, CI [-.28, .28], Cohen's $q=.00)$ and Agreeableness $(z=1.25, p=.210$, CI $[-.11, .46]$, Cohen's $q=.182)$. The results thus suggest that participants assumed their partner to have higher similarity in Honesty-Humility compared to all 
ASSUMED PERSONALITY SIMILARITY IN COUPLES

the other HEXACO traits (even higher than Openness, $\mathrm{z}=2.63, p=.009$, CI [.08, .57], Cohen's $q=.378)$, and assumed higher similarity in Openness compared to Emotionality and Conscientiousness, but not to Extraversion and Agreeableness. Consequently, the findings supported Hypothesis 1, and partially supported Hypothesis 2.

However, the study had some limitations. Most importantly, only one member of an intimate dyad participated. Thus, what we have labelled as higher assumed similarity in HonestyHumility and (partly) Openness might in fact be due to higher actual similarity in these two traits. Since the person who provided data on their own personality was our only source of information on the personality of the partner, we cannot exclude this alternative explanation. One strategy to control for actual similarity would be to include both members of an intimate relationship (each member providing a self- and a partner report).

Another limitation of Study 1 was the relatively small sample size. Definitely, a larger sample could further validate the robustness of our findings. Additionally, relationship length may have an influence on assumed similarity in personality between intimate partners. Possibly, people who are in a longer relationship with their partner are more accurate in their evaluation of their partners' personality (reducing assumed similarity). However, Study 1 included no assessment of relationship length. In Study 2, we addressed these three limitations.

\section{Study 2: Chinese Study}

\section{Participants and Procedure}

Participants were recruited through flyers and online advertisement from a university in the northwest of China. To be eligible for the current study, participants had to be in a heterosexual relationship for minimum 6 months and have no children. 118 intimate dyads $(N=$ 236 individuals) meeting these requirements attended our research in a lab. Both partners 
ASSUMED PERSONALITY SIMILARITY IN COUPLES

completed all the assessments in two separate tables back to back, and the order of the questionnaires (i.e., whether the self- or partner report was administered first) was randomly assigned. Participants were instructed to complete the surveys without discussion, and were paid and debriefed upon completion. Participants were on average $22.17(S D=2.65)$ years old, and their relationship length ranged from 6 to $87(M=18.93, S D=16.10)$ months.

As with Study 1, we sought to enroll every participant we could get within a reasonable time frame. However, the results of Study 1 are useful for helping us to evaluate the power adequacy of this sample. That is, we did a pre-power analysis for the required sample size for Study 2 based on the results from Study 1. Specifically, we assessed the sample size needed to detect a significant difference between Honesty-Humility $\left(r_{I C C}=.50\right)$ and the largest correlation among the other four traits in Study 1, in this case, Extraversion $\left(r_{I C C}=.17\right)$, which enables us to have a large effect size with Cohen's $q$ equaling to .378 . To reach the power $1-\beta=.95$ at a significant level of .05 , the required sample is 185 . So, we aimed to have this sample size as a minimum for this study, while again continuing recruiting as long as our time and financial resources allowed us to do so.

\section{Measures}

We used the Chinese (i.e., Mandarin) version of the same measures administered in Study 1. The HEXACO items had already been translated into Chinese before (see www.hexaco.org). However, we adapted a few items slightly to further improve their readability and clarity based on the recommendations from two bilingual (Chinese-English) psychologists.

\section{Results and Discussion}

The upper right of Table 1 shows the similarity correlations for the six personality traits between self- and partner ratings in our Chinese sample. As with Study 1, we compared the ICCs 
ASSUMED PERSONALITY SIMILARITY IN COUPLES

of Honesty-Humility and Openness with these of the other four traits and report specific $p$-values for all tests. The ICC of Honesty-Humility $\left(r_{I C C}=.46\right)$ was significantly higher than the ICCs of Emotionality $(z=8.04, p<.001$, CI $[.49, .80]$, Cohen's $q=.690)$, Extraversion $(z=4.51, p<$ $.001, \mathrm{CI}[.21, .53]$, Cohen's $q=.407)$, Agreeableness $(z=4.80, p<.001$, CI $[.22, .53]$, Cohen's $q$ $=.417)$, and Conscientiousness $(z=5.73, p<.001$, CI $[.31, .63]$, Cohen's $q=.507)$. However, the ICC of Openness $\left(r_{I C C}=.21\right)$ was significantly higher than the ICCs of Emotionality $(z=$ 4.53, $p<.001$, CI $[.22, .57]$, Cohen's $q=.406)$ and (depending on the $p$-threshold) Conscientiousness $(z=2.49, p=.013$, CI $[.04, .39]$, Cohen's $q=.223)$ only, but was not higher than the ICCs of Extraversion $(z=1.35, p=.177$, CI $[-.06, .29]$, Cohen's $q=.123)$ and Agreeableness $(z=1.48, p=.139$, CI [-.04, .30], Cohen's $q=.133)$. Further, the ICC of HonestyHumility was also higher than the ICC of Openness, $\mathrm{z}=3.12, p=.002$, CI $[.09, .41]$, Cohen's $q$ $=.284)$. The results completely replicated the findings from Study 1, again supporting Hypothesis 1, and only partially supporting Hypothesis 2. Additionally, compared to the assumed similarity, the ICCs of actual similarity between couples in the HEXACO traits were rather small, ranging from -.15 to .07 , with -.04 for Honesty-Humility and .07 for Openness (see Table 2).

Next, we tested whether assumed similarity in Honesty-Humility and partly Openness was still higher than the assumed similarity in the remaining HEXACO traits after controlling for actual similarity within dyads. To control for the actual similarity, we performed multilevel modeling which allows the intercepts of the two individuals within one dyad to covary. Using the lme function from the nlme package in R (Pinheiro, Bates, DebRoy, Sarkar and R Core Team, 2017), we conducted six separate multilevel models for each of the HEXACO trait, using one's self-report personality and one's partner's self-report personality to predict one's perception of 
ASSUMED PERSONALITY SIMILARITY IN COUPLES

one's partner's personality. As illustrated in Figure 1, assumed similarity is obtained by using one's self-report personality to predict one's perception of one's partner's personality, accurate perception is obtained by using one's partner's self-report personality to predict one's perception of one's partner's personality, and actual similarity is controlled by the multilevel modeling.

All the multilevel analyses were performed controlling for relationship length and age.

Results are presented in Table 3, indicating that the assumed similarity in Honesty-Humility was significantly higher than the ones in Emotionality $(z=7.96, p<.001)$, Extraversion $(z=5.14, p<$ $.001)$, Agreeableness $(z=4.81, p<.001)$, and Conscientiousness $(z=5.68, p<.001)$. However, again, assumed similarity in Openness was only significantly higher than the ones in Emotionality $(z=4.85, p<.001)$, Conscientiousness $(z=2.57, p=.010$; depending on the $p$ threshold) and Extraversion $(z=2.02, p=.043)$, but not Agreeableness $(z=1.69, p=.090)$. Interestingly, we also found that people's perceptions of their partner's Honesty-Humility $(b=$ .28) was significantly less accurate than their perception of their partner's Openness $(b=.47), z$ $=3.12, p=.002$. Thus, it seems that people have a more accurate judgement of their partners' Openness as compared to Honesty-Humility.

In addition, we also examined whether relationship length moderated the relation between one's self-report personality and one's observer report about one's partner's personality (i.e., assumed similarity). One reason to perform these analyses was that assumed similarity in Honesty-Humility and Openness might be lower for people who have a longer relationship because they have had more opportunities to observe their partners, and, in turn, to make more accurate judgments. To test this idea, we predicted one's perception of one's partner's HonestyHumility from one's self-report Honesty-Humility, one's partner's self-report Honesty-Humility, relationship length, and an interaction term between one's self-report Honesty-Humility and 
ASSUMED PERSONALITY SIMILARITY IN COUPLES

relationship length. We performed another parallel analysis, investigating the interaction between relationship length and Openness. The results showed that neither the interaction between Honesty-Humility and relationship length $(b=.004, p=.357)$ nor the interaction between Openness and relationship length $(b=-.001, p=.890)$ was significant. Thus, relationship length did not moderate the effect of assumed similarity in Honesty-Humility and Openness. ${ }^{\text {iv }}$

In sum, people showed higher assumed similarity in Honesty-Humility compared to all the other personality traits, including Openness, and in Openness only with regard to Emotionality and (potentially) Conscientiousness. These results still held even after controlling for age, relationship length, actual similarity, and perception accuracy.

\section{Supplementary Analyses}

The presented two studies were conducted in two different countries, China and Denmark, providing us a chance to examine potential differences in assumed similarity between these countries. Specifically, it has been suggested that personality traits are particularly salient in North American and European populations: compared to East Asian populations, members of Western populations are more prone to use personality trait terms to describe themselves, are faster to indicate a trait attribute as a descriptor of them, and are more prone to retain personality traits as highly cognitively accessible (Markus \& Kitayama, 1998). This might be true concerning intimate relationships in particular. For example, when asked about the reasons for getting married, Chinese couples tended to play stronger emphasis on factors outside the intimate relationship, such as their relationship with in-laws and friends, and the approval of their family (Chang \& Chan, 2007), suggesting a relatively unimportant role of personality for Chinese couples. Also, a study by Buss (1990) across 37 cultures indicated that Chinese people 
ASSUMED PERSONALITY SIMILARITY IN COUPLES

emphasized the personality of their desired partners less as compared to their western counterparts.

In this light, one might expect that the assumed similarity in Honesty-Humility and Openness may be lower in the Chinese sample compared to the Danish sample because a Chinese population might generally place less emphasis on their partners' personality and, in turn, might have a lower motivation to assume similarity in personality with their partners. Importantly, however, given the differences of the two studies concerning the design (online vs in the lab), the number of participants, and the diversity of the participants (one member of a couple vs both members of a couple; students from introductory courses in psychology vs students from different fields), we tested any differences between the two samples (i.e., countries) in an explorative manner only.

We compared the ICCs of Honesty-Humility ( $r=.50$ for Denmark and $r=.46$ for China) and Openness ( $r=.17$ for Denmark and $r=.21$ for China) from the two countries with the method of comparing two correlations from two independent samples using the cocor package in R (Diedenhofen \& Musch, 2015). The results indicate that there was no difference in assumed similarity in Honesty-Humility $(z=.42, p=.675, C I[-.15, .22])$ and Openness $(z=-.33, p=$ $.738, C I[-.28, .19])$ between China and Denmark, suggesting that participants from both samples tended to assume higher similarity in Honesty-Humility and Openness in a similar degree. ${ }^{v}$

\section{General Discussion}

The current research examined whether people in intimate relationships in China and Denmark assumed higher similarity between themselves and their partner in Honesty-Humility and Openness compared to the other four HEXACO traits. The results supported the idea that people assume their intimate partner to have higher similarity in Honesty-Humility, and partially 
ASSUMED PERSONALITY SIMILARITY IN COUPLES

supported the idea that people assume higher similarity in Openness compared to the other four traits. In the Chinese sample we found these findings persisted even after controlling for age, relationship length, and the partner's self-report of their personality, indicating that it is truly assumed and not actual similarity that is elevated for these traits (partly for Openness). The rigorous demonstration of true assumed similarity in the Chinese sample represents a particularly noteworthy result, given that the comparatively lower emphasis on personality traits in East Asian cultures observed in previous work (Markus \& Kitayama, 1998) pointed to the possibility that existing findings on assumed similarity might not generalize to non-Western contexts. However, the present results suggest that even though people in non-individualistic cultures might understand themselves and their acquaintances less in terms of personality than in terms of context and relationships, assumptions of personality similarity remain present and substantial across cultural contexts. However, as mentioned above, we only tested the potential cultural differences in an exploratory way, and future research might continue exploring whether couples tend to assume higher similarity in Honesty-Humility and Openness across cultures more thoroughly (i.e., using more comparable methods, samples and research designs).

Our findings focusing on intimate dyads are generally consistent with the findings reported by Lee et al. (2009) and Cohen et al. (2013) considering well-acquainted dyads, such as friends and co-workers. Importantly, our studies not only extended prior research relying on mixed samples, but also statistically tested the robustness of the conclusion that assumed similarity is indeed higher in Honesty-Humility and Openness. In sum, the current research suggests that people tend to assume higher similarity with their intimate partners regarding Honesty-Humility and Openness (partially). 
ASSUMED PERSONALITY SIMILARITY IN COUPLES

The tendency for intimate partners to assume higher similarity in Honesty-Humility and Openness (partially) — despite the possibility of achieving more accurate judgements due to more available information — strongly implicates the importance of assumed similarity in these two traits for intimate relationships. According to motivated cognition (Kunda, 1990; Lemay \& Clark, 2015), people are motivated to see their partners in a particular way in order to achieve their desired relationship outcomes. Although, to the best of our knowledge, no study has directly investigated the role of assumed similarity in Honesty-Humility and Openness for relationship outcomes, some indirect support for such a link can be found. Tidwell, Eastwick, and Finkel (2013) found that assumed similarity in Dependable/Trustworthiness and Intellectually Sharp, rather than actual similarity, in a potential partner predicted people's romantic attraction towards that partner. As Dependable/Trustworthiness and Intellectually Sharp are conceptually related to Honesty-Humility and Openness (Lee \& Ashton, 2004, 2006), it seems likely that assumed similarity in these two traits predicts initial attraction or relationship formation. Additionally, the higher motivation to assumed similarity in Honesty-Humility and Openness might also implicate the importance of these two traits for relationship outcomes. Future research could examine these ideas.

The lower and less consistent motivation to assume similarity in the other HEXACO trait, Emotionality, Extraversion, Agreeableness, and Conscientiousness, might imply that assumed similarity in these traits is less important for relationship outcomes. Instead of assuming similarity, people might develop other motivations to facilitate their relationship, such as assuming lower or higher levels of these traits in their partners. For example, people might be motivated to assume their partners to have lower levels of Neuroticism, and higher levels of Extraversion, Agreeableness, and Conscientiousness since having partners with these 
ASSUMED PERSONALITY SIMILARITY IN COUPLES

characteristics contributes to people's relationship satisfaction (Malouff, Thorsteinsson, Schutte, Bhullar, \& Rooke, 2010).

The current study also found that assumed similarity in Openness is relatively lower and less consistent compared with assumed similarity in Honesty-Humility. There are two potential explanations for this, one concerning the visibility of the trait (as measured by the corresponding items), and the other one concerning the importance of the trait for intimate relationships. Regarding the former, it has been suggested that for traits with high visibility (i.e., those with clear and frequent behavioral manifestations), accuracy in personality judgments tends to be higher (McAbee \& Connelly 2016; Vazire, 2010). Thus, the lower assumed similarity in Openness might be explained by the higher visibility of the items assessing Openness, compared to the items assessing Honesty-Humility. Actually, 7 out of 10 items assessing Openness in the HEXACO-60 seem relatively easy to observe for intimate partners (e.g., "I would enjoy creating a work of art, such as a novel, a song, or a painting,", "I find it boring to discuss philosophy"). Correspondingly, in Study 2, we found higher accuracy $(b=.48)$, and potentially lower assumed similarity $(b=.28)$, in Openness, while the pattern was opposite for Honesty-Humility $(b=.52$ for assumed similarity and $b=.28$ for accuracy).

Regarding the potential importance of the traits for intimate relationships, the lower assumed similarity in Openness might be due to the fact that assumed similarity in Openness is less important for intimate relationships than assumed similarity in Honesty-Humility. Though no study has compared the significance of assumed similarity in Honesty-Humility and Openness on relationship outcomes directly, some indirect support for such a difference can be found. For example, Schaffhuser, Allemand, Werner, and Martin (2016) found that assumed similarity in Big Five Agreeableness (somewhat, though not too strongly, overlapping with Honesty-Humility) 
ASSUMED PERSONALITY SIMILARITY IN COUPLES

was positively related to relationship satisfaction whereas assumed similarity in Openness

(similar to HEXACO-Openness) was not. Consequently, people might be more motivated to assumed higher similarity in Honesty-Humility than in Openness. However, these are just initial thoughts which need further examination in the future.

Future research might also explore the underlying mechanism of assumed similarity in couples. As shown by prior research, Honesty-Humility and Openness are closely linked to various personal attitudes and values relative to the other four traits (e.g., Lee et al., 2009; Liu et al., 2017; Liu, Zettler, \& Hilbig, 2016). Based on this, future studies might explore whether important personal attitudes and/or values mediate assumed similarity in Honesty-Humility and Openness between intimate partners. Additionally, although the current research found no moderation effects of relationship length on assumed similarity in Honesty-Humility and Openness within intimate dyads, future studies could continue exploring potential moderators, such as closeness. Perhaps assumed similarity is higher for couples who share great intimacy since they have greater motivation for such a bias to enhance relationship outcomes. Finally, it seems important to examine the development of assumed similarity in Honesty-Humility and Openness over time. As relationships develop, assumed similarity in Honesty-Humility or Openness might also change, such as remaining stable, increasing, or decreasing, due to changes in placing importance on different relationship features (e.g., when couples start to bring up children).

Despite the conceptually similar findings between Denmark and China, there are several limitations of our investigation. First, participants were primarily college students, so that future research may want to examine the current effects in more diverse samples. Second, the relatively short time interval between self-report and partner report personality may have led to potential 
ASSUMED PERSONALITY SIMILARITY IN COUPLES

carry-over effects. Thus, the answering patterns in the first questionnaire may have exerted some influences on the answering patterns in the second questionnaire (although we aimed to mitigate such effects by randomizing the order of the questionnaires in Study 2).

\section{Conclusions}

The current research investigated whether intimate partners in Denmark and China assumed higher similarity with each other in Honesty-Humility and Openness compared to the other four traits of the HEXACO Model of Personality (i.e., Emotionality, Extraversion, Agreeableness and Consciousness). The results suggest that people assume their intimate partner to have higher similarity in Honesty-Humility and Openness (partially) compared to the other four traits, and that such preferences are not different across the two countries. Overall, the current research implicates the importance of assumed similarity in Honesty-Humility and Openness for intimate relationships, and, more generally, points at differences in the degree of assumed similarity between different traits. 
ASSUMED PERSONALITY SIMILARITY IN COUPLES

Acknowledgements:

The research was partially supported by a grant from the Asian Dynamics Initiatives, University of Copenhagen, Denmark, to the first author.

All authors contributed to designing studies, acquiring data, interpreting data, as well as editing the manuscript. J.L performed the analyses and prepared the manuscript. 
ASSUMED PERSONALITY SIMILARITY IN COUPLES

References

Ashton, M. C., \& Lee, K. (2005). Honesty-Humility, the Big Five, and the Five-Factor Model. Journal of Personality, 73(5), 1321-1354. https://doi.org/10.1111/j.14676494.2005.00351.x

Ashton, M. C., \& Lee, K. (2007). Empirical, theoretical, and practical advantages of the HEXACO model of personality structure. Personality and Social Psychology Review, 11(2), 150-166. https://doi.org/10.1177/1088868306294907

Ashton, M. C., \& Lee, K. (2009). The HEXACO-60: A short measure of the major dimensions of personality. Journal of Personality Assessment, 91(4), 340-345. https://doi.org/10.1080/00223890902935878

Ashton, M. C., Lee, K., \& De Vries, R. E. (2014). The HEXACO Honesty-Humility, Agreeableness, and Emotionality factors: A review of research and theory. Personality and Social Psychology Review, 18(2), 139-152.

Bradbury, T. N., \& Karney, B. R. (2010). Intimate relationships. New York, NY: W. W. Norton.

Buss. D. M. (1990). International preferences in selecting mates: A study of 37 cultures. Journal of Cross-Cultural Psychology, 21, 5-47.

Byrne, D. (1971). The attraction paradigm. New York, NY: Academic Press.

Chang, S. C., \& Chan, C. N. (2007). Perceptions of commitment change during mate selection: The case of Taiwanese newlyweds. Journal of Social and Personal Relationships, 24(1), 55-68. https://doi.org/10.1177/0265407507072583

Cohen, T. R., Panter, A. T., Turan, N., Morse, L., \& Kim, Y. (2013). Agreement and similarity in self-other perceptions of moral character. Journal of Research in Personality, 47(6), 816830. https://doi.org/10.1016/j.jrp.2013.08.009 
ASSUMED PERSONALITY SIMILARITY IN COUPLES

Diedenhofen, B., \& Musch, J. (2015). cocor: A comprehensive solution for the statistical comparison of correlations. PLOS ONE, 10(4), e0121945. https://doi.org/10.1371/journal.pone.0121945

Furr, R. M. (2010). The double-entry intraclass correlation as an index of profile similarity: meaning, limitations, and alternatives. Journal of Personality Assessment, 92(1), 1-15. https://doi.org/10.1080/00223890903379134

Johnson, D. J., \& Rusbult, C. E. (1989). Resisting temptation: Devaluation of alternative partners as a means of maintaining commitment in close relationships. Journal of Personality and Social Psychology, 57(6), 967.

Kenny, D. A., \& West, T. V. (2010). Similarity and agreement in self-and other perception: A meta-analysis. Personality and Social Psychology Review, 14(2), 196-213. https://doi.org/10.1177/1088868309353414

Kenny, D. A., \& Acitelli, L. K. (2001). Accuracy and bias in the perception of the partner in a close relationship. Journal of Personality and Social Psychology, 80(3), 439-448. https://doi.org/10.1037/0022-3514.80.3.439

Kunda, Z. (1990). The case for motivated reasoning. Psychological Bulletin, 108(3), 480.

Lee, K., \& Ashton, M. C. (2004). Psychometric properties of the HEXACO personality inventory. Multivariate Behavioral Research, 39(2), 329-358. https://doi.org/10.1207/s15327906mbr3902_8

Lee, K., \& Ashton, M. C. (2006). Further assessment of the HEXACO Personality Inventory: Two new facet scales and an observer report form. Psychological Assessment, 18(2), 182-191. https://doi.org/10.1037/1040-3590.18.2.182 
ASSUMED PERSONALITY SIMILARITY IN COUPLES

Lee, K., Ashton, M. C., Pozzebon, J. A., Visser, B. A., Bourdage, J. S., \& Ogunfowora, B. (2009). Similarity and assumed similarity in personality reports of well-acquainted persons. Journal of Personality and Social Psychology, 96(2), 460-472. https://doi.org/10.1037/a0014059

Lemay, E. P., \& Clark, M. S. (2015). Motivated cognition in relationships. Current Opinion in Psychology, 1, 72-75. https://doi.org/10.1016/j.copsyc.2014.11.002

Lemay, E. P., \& Neal, A. M. (2014). Accurate and biased perceptions of responsive support predict well-being. Motivation and Emotion, 38(2), 270-286. https://doi.org/10.1007/s11031-013-9381-2

Liu, J., Ludeke, S. G., \& Zettler, I. (2017). The HEXACO correlates of authoritarianism's facets in the U.S. and Denmark. Personality and Individual Differences, 116, 348-352. https://doi.org/10.1016/j.paid.2017.05.015

Liu, J., Ludeke, S. G., Haubrich, J., Gondan, M., \& Zettler, I. (2018). Similar to and/or better thanoneself? Singles' ideal partner personality descriptions. Manuscript submitted for publication.

Liu, J., Zettler, I., \& Hilbig, B. E. (2016). Honesty-Humility. In V. Zeigler-Hill \& T. K. Shackelford (Eds.), Encyclopedia of Personality and Individual Differences (pp. 1-9). Cham: Springer International Publishing.

Malouff, J. M., Thorsteinsson, E. B., Schutte, N. S., Bhullar, N., \& Rooke, S. E. (2010). The five-factor model of personality and relationship satisfaction of intimate partners: A meta-analysis. Journal of Research in Personality, 44(1), 124-127. https://doi.org/10.1016/j.jrp.2009.09.004 
ASSUMED PERSONALITY SIMILARITY IN COUPLES

Maner, J. K., Gailliot, M. T., \& Miller, S. L. (2009). The implicit cognition of relationship maintenance: Inattention to attractive alternatives. Journal of Experimental Social Psychology, 45(1), 174-179. https://doi.org/10.1016/j.jesp.2008.08.002

Markus, H. R., \& Kitayama, S. (1998). The cultural psychology of personality. Journal of CrossCultural Psychology, 1(1998), 63-87. https://doi.org/0803973233

McAbee, T. S., \& Connelly, S. B. (2016). A multi-rater framework for studying personality: The trait-reputation-identity model. Psychological Review, 123(5), 569 -591.

McCrae, R. R., Martin, T. A., Hrebícková, M., Urbánek, T., Boomsma, D. I., Willemsen, G., \& Costa, P. T. (2008). Personality trait similarity between spouses in four cultures. Journal of Personality, 76(5), 1137-1164. https://doi.org/10.1111/j.1467-6494.2008.00517.x

Montoya, R. M., Horton, R. S., \& Kirchner, J. (2008). Is actual similarity necessary for attraction? A meta-analysis of actual and perceived similarity. Journal of Social and Personal Relationships, 25(6), 889-922. https://doi.org/10.1177/0265407508096700

Murray, S. L., Holmes, J. G., Bellavia, G., Griffin, D. W., \& Dolderman, D. (2002). Kindred spirits? The benefits of egocentrism in close relationships. Journal of Personality and Social Psychology, 82(4), 563-581. https://doi.org/10.1037//0022-3514.82.4.563

Murray, S. L., Holmes, J. G., Dolderman, D., \& Griffin, D. W. (2000). What the motivated mind sees: Comparing friends' perspectives to married partners' views of each other. Journal of Experimental Social Psychology, 36(6), 600-620. https://doi.org/10.1006/jesp.1999.1417

Paunonen, S. V., \& Hong, R. Y. (2013). The many faces of assumed similarity in perceptions of personality. Journal of Research in Personality, 47(6), 800-815. https://doi.org/10.1016/j.jrp.2013.08.007 
ASSUMED PERSONALITY SIMILARITY IN COUPLES

Pinheiro, J., Bates, D., DebRoy, S., Sarkar, D., and R Core Team (2017). nlme: Linear and Nonlinear Mixed Effects Models. R package version 3.1-131, https:/CRAN.Rproject.org/package $=$ nlme.

Preisler, B. (2003). English in Danish and the Danes' English. International Journal of the Sociology of Language, (159), 109-126.

Schaffhuser, K., Allemand, M., Werner, C. S., \& Martin, M. (2016). Discrepancy in personality perceptions is related to relationship satisfaction: Findings from dyadic latent discrepancy analyses. Journal of Personality, 84(5), 658-670. https://doi.org/10.1111/jopy.12189

Srivastava, S., Guglielmo, S., \& Beer, J. S. (2010). Perceiving others' personalities: Examining the dimensionality, assumed similarity to the self, and stability of perceiver effects. Journal of Personality and Social Psychology, 98(3), 520-534. https://doi.org/10.1037/a0017057

Tidwell, N. D., Eastwick, P. W., \& Finkel, E. J. (2013). Perceived, not actual, similarity predicts initial attraction in a live romantic context: Evidence from the speed-dating paradigm. Personal Relationships, 20(2), 199-215. https://doi.org/10.1111/j.14756811.2012.01405.x

Vazire, S. (2010). Who knows what about a person? The self-other knowledge asymmetry (SOKA) model. Journal of Personality and Social Psychology, 98(2), 281-300. https://doi.org/10.1037/a0017908

Watson, D., Hubbard, B., \& Wiese, D. (2000). Self-other agreement in personality and affectivity: The role of acquaintanceship, trait visibility, and assumed similarity. Journal of Personality and Social Psychology, 78(3), 546-558. https://doi.org/10.1037//00223514.78.3.546 


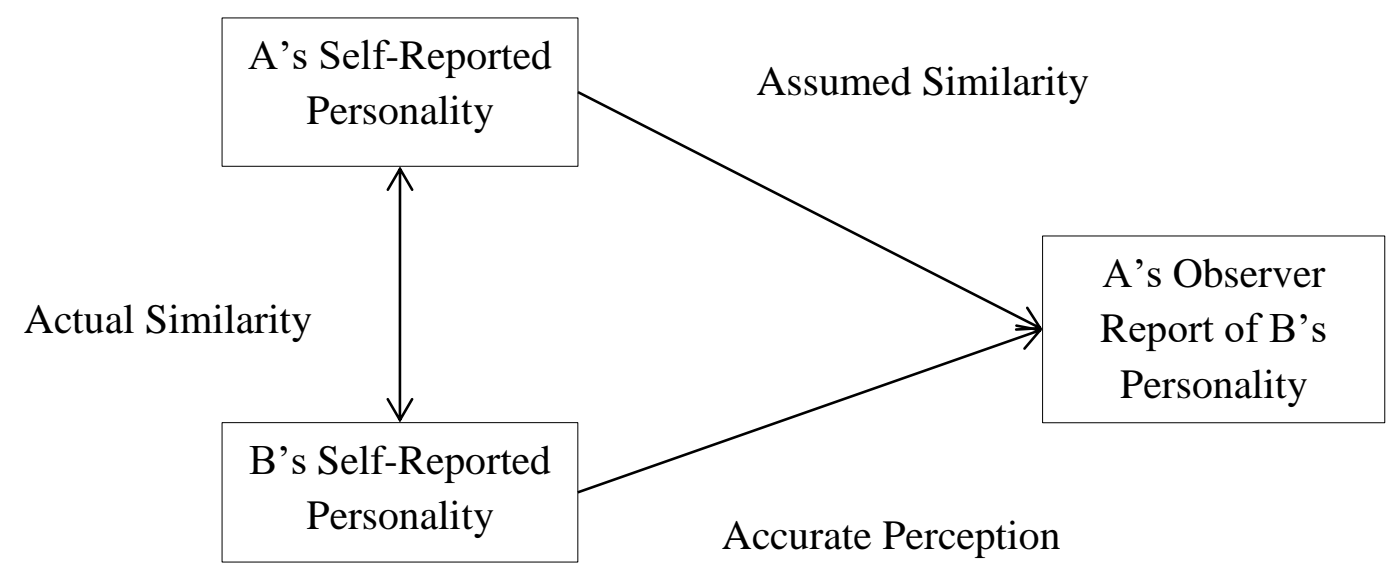

Figure 1. Relations between assumed similarity, actual similarity, and accurate perception in a partnership of Person A and Person B. Assumed similarity is assessed using A's self-report personality to predict A's observer report of B's personality, accurate perception is assessed using B's self-report personality to predict A's observer report of B's personality, and the actual similarity is the correlation between A's and B's self-reports of the same personality characteristic. 


\section{ASSUMED PERSONALITY SIMILARITY IN COUPLES}

Table 1

Means, Standard Deviations, Cronbach Alphas, and Correlations Between Self- and Partner Ratings for Study 1 and Study 2

\begin{tabular}{|c|c|c|c|c|c|c|c|c|c|c|c|c|c|c|}
\hline & \multicolumn{2}{|c|}{$M(S D)$} & \multirow[t]{2}{*}{1} & \multirow[t]{2}{*}{2} & \multirow[t]{2}{*}{3} & \multirow[t]{2}{*}{4} & \multirow[t]{2}{*}{5} & \multirow[t]{2}{*}{6} & \multirow[t]{2}{*}{7} & \multirow[t]{2}{*}{8} & \multirow[t]{2}{*}{9} & \multirow[t]{2}{*}{10} & \multirow[t]{2}{*}{11} & \multirow[t]{2}{*}{12} \\
\hline & DK & $\mathrm{CN}$ & & & & & & & & & & & & \\
\hline 1. SR-H & $\begin{array}{l}3.55 \\
(.65)\end{array}$ & $\begin{array}{l}3.36 \\
(.56)\end{array}$ & $.80 / .66$ & -.06 & -.02 & $.29 *$ & .09 & $.11 *$ & $.46^{*}$ & -.05 & $.07 *$ & $.13^{*}$ & $.14^{*}$ & .07 \\
\hline 2. SR-E & $\begin{array}{l}3.37 \\
(.66)\end{array}$ & $\begin{array}{l}3.38 \\
(.54)\end{array}$ & .13 & $.82 / .66$ & $-.14^{*}$ & $-.20^{*}$ & .02 & -.01 & -.05 & $-.19 *$ & -.05 & $.10^{*}$ & -.03 & .03 \\
\hline 3. SR-X & $\begin{array}{l}3.49 \\
(.71)\end{array}$ & $\begin{array}{l}3.50 \\
(.53)\end{array}$ & -.06 & -.13 & $.83 / .70$ & $.26^{*}$ & $.18^{*}$ & $.14^{*}$ & .07 & -.05 & .09 & $.13^{*}$ & $.14^{*}$ & .02 \\
\hline 4. SR-A & $\begin{array}{l}3.23 \\
(.59)\end{array}$ & $\begin{array}{l}3.44 \\
(.47)\end{array}$ & $.42 *$ & .00 & .02 & $.86 / .60$ & $.14^{*}$ & $.20 *$ & $.13^{*}$ & $.10^{*}$ & $.13 *$ & .08 & $.16^{*}$ & $.13 *$ \\
\hline 5. SR-C & $\begin{array}{l}3.73 \\
(.63)\end{array}$ & $\begin{array}{l}3.43 \\
(.51)\end{array}$ & $.19 *$ & $.32 *$ & .05 & .07 & $.84 / .68$ & $.23 *$ & $.14^{*}$ & -.03 & $.14 *$ & $.16^{*}$ & -.01 & $.10 *$ \\
\hline 6. SR-O & $\begin{array}{l}3.70 \\
(.55)\end{array}$ & $\begin{array}{l}3.40 \\
(.55)\end{array}$ & $.15^{*}$ & .13 & .04 & .10 & -.05 & $.80 / .63$ & .07 & .03 & .02 & $.13^{*}$ & $.10 *$ & $.21 *$ \\
\hline 7. CP-H & $\begin{array}{l}3.55 \\
(.66)\end{array}$ & $\begin{array}{l}3.46 \\
(.60)\end{array}$ & $.50 *$ & -.08 & -.01 & $.19 *$ & .10 & .01 & $.79 / .70$ & -.06 & -.02 & $.29 *$ & .09 & $.11^{*}$ \\
\hline 8. CP-E & $\begin{array}{l}2.85 \\
(.66)\end{array}$ & $\begin{array}{l}3.35 \\
(.68)\end{array}$ & -.08 & $-.29 *$ & .07 & .03 & $-.21 *$ & -.08 & .13 & $.84 / .80$ & $-.14^{*}$ & $-.20 *$ & .02 & -.01 \\
\hline 9. CP-X & $\begin{array}{l}3.52 \\
(.60)\end{array}$ & $\begin{array}{l}3.54 \\
(.61)\end{array}$ & -.01 & .07 & $.17 *$ & .14 & .00 & $.15^{*}$ & -.06 & -.13 & $.87 / .78$ & $.26^{*}$ & $.18 *$ & $.14^{*}$ \\
\hline 10. CP-A & $\begin{array}{l}3.26 \\
(.75)\end{array}$ & $\begin{array}{l}3.65 \\
(.61)\end{array}$ & $.19 *$ & .03 & .14 & -.01 & .14 & -.03 & $.42 *$ & .00 & .02 & $.75 / .78$ & $.14^{*}$ & $.20 *$ \\
\hline 11. CP-C & $\begin{array}{l}3.38 \\
(.72)\end{array}$ & $\begin{array}{l}3.31 \\
(.66)\end{array}$ & .10 & $-.21 *$ & -.00 & .14 & $-.25 *$ & -.05 & $.19^{*}$ & $.32 *$ & .05 & .07 & $.82 / .79$ & $.23^{*}$ \\
\hline
\end{tabular}


ASSUMED PERSONALITY SIMILARITY IN COUPLES

\begin{tabular}{llllllllllllllll}
12. & $\mathrm{CP}-\mathrm{O}$ & 3.23 & 3.15 & .01 & -.08 & $.15^{*}$ & -.03 & -.05 & $.17 *$ & $.15^{*}$ & .13 & .04 & .10 & -.05 & $.76 / .74$ \\
\hline & $(.70)$ & $(.63)$ & & &
\end{tabular}

Note. $\mathrm{SR}=$ self-ratings, $\mathrm{CP}=$ current partner ratings, $\mathrm{DK}=$ Denmark, $\mathrm{CN}=\mathrm{China}, \mathrm{H}=$ Honesty-Humility, $\mathrm{E}=$ Emotionality, $\mathrm{X}=$

Extraversion, $\mathrm{A}=$ Agreeableness, $\mathrm{C}=$ Conscientiousness, $\mathrm{O}=$ Openness to Experience. Correlations below the diagonal are for

Denmark, and correlations above the diagonal are for China. ICCs for the same trait are printed in bold. Cronbach's alphas are presented in the diagonal, in the format of Denmark/China. The sample size is $N=93$ for Denmark and $N=236$ for China.

$* p<.05$. 


\section{ASSUMED PERSONALITY SIMILARITY IN COUPLES}

Table 2

ICCs of the Self-Reports in the HEXACO Traits for the Chinese Couples (Study 2)

\begin{tabular}{|c|c|c|c|c|c|c|c|c|c|c|c|}
\hline Variable & 1. & 2. & 3. & 4. & 5. & 6. & 7. & 8. & 9. & 10. & 11. \\
\hline \multicolumn{12}{|l|}{ 1. F-H } \\
\hline 2. F-E & -.04 & & & & & & & & & & \\
\hline 3. F-X & -.04 & -.08 & & & & & & & & & \\
\hline 4. F-A & $.19^{*}$ & $-.14 *$ & $.24 *$ & & & & & & & & \\
\hline 5. F-C & $.17 *$ & -.06 & $.30 *$ & $.26^{*}$ & & & & & & & \\
\hline 6. $\mathrm{F}-\mathrm{O}$ & .08 & -.05 & $.13^{*}$ & $.26^{*}$ & $.19 *$ & & & & & & \\
\hline 7. M-H & -.04 & -.08 & -.08 & .02 & -.08 & -.02 & & & & & \\
\hline 8. M-E & -.08 & $-.15 *$ & $-.13 *$ & -.04 & -.01 & -.06 & -.04 & & & & \\
\hline 9. M-X & -.08 & $-.13 *$ & .01 & .03 & .02 & $-.14 *$ & -.04 & -.08 & & & \\
\hline 10. M-A & .02 & -.04 & .03 & .05 & -.04 & .10 & $.19 *$ & $-.14 *$ & $.24 *$ & & \\
\hline 11. M-C & -.08 & -.01 & .02 & -.04 & -.08 & -.05 & $.17 *$ & -.06 & $.30 *$ & $.26^{*}$ & \\
\hline 12. M-O & -.02 & -.06 & $-.14 *$ & .10 & -.05 & .07 & .08 & -.05 & $.13^{*}$ & $.26^{*}$ & $.19^{*}$ \\
\hline
\end{tabular}

Note. $N=118 . \mathrm{F}=$ female, $\mathrm{M}=$ male $\mathrm{H}=$ Honesty-Humility, $\mathrm{E}=$ Emotionality, $\mathrm{X}=$ Extraversion, $\mathrm{A}=\mathrm{Agreeableness}, \mathrm{C}=$

Conscientiousness, $\mathrm{O}=$ Openness to Experience. ICCs for the same traits (i.e., actual similarity) are printed in bold.

$* p<.05$ 


\section{ASSUMED PERSONALITY SIMILARITY IN COUPLES}

Table 3

Results of the Analyses Predicting Perception of One's Partner's Personality Based on Self-and Partner Ratings

Controlling for Age and Relationship Length (Study 2)

\begin{tabular}{ccccccccccc}
\hline Traits & \multicolumn{1}{c}{ Self-report } \\
\hline & $b$ & $\beta$ & $S E$ & $t$ & $95 \% C I$ & $b$ & $\beta$ & $S E$ & $t$ & $95 \% C I$ \\
$\mathrm{H}$ & $.52^{*}$ & .29 & .06 & 8.86 & {$[.41, .64]$} & $.28^{*}$ & .16 & .06 & 4.82 & {$[.17, .40]$} \\
$\mathrm{E}$ & $-.16^{*}$ & -.09 & .07 & -2.23 & {$[-.30,-.02]$} & $.65^{*}$ & .35 & .07 & 9.15 & {$[.51, .79]$} \\
$\mathrm{X}$ & .10 & .05 & .07 & 1.46 & {$[-.04, .24]$} & $.39^{*}$ & .20 & .07 & 5.47 & {$[.25, .53]$} \\
$\mathrm{A}$ & .13 & .06 & .08 & 1.68 & {$[-.02, .29]$} & $.42^{*}$ & .20 & .08 & 5.33 & {$[.27, .58]$} \\
$\mathrm{C}$ & .05 & .02 & .08 & .61 & {$[-.10, .20]$} & $.55^{*}$ & .28 & .08 & 7.05 & {$[.40, .70]$} \\
$\mathrm{O}$ & $.28 *$ & .15 & .07 & 4.23 & {$[.15, .41]$} & $.48^{*}$ & .26 & .07 & 7.25 & {$[.35, .60]$} \\
\hline &
\end{tabular}


${ }^{\mathrm{i}}$ The same participant pool has been used for another two manuscripts [Liu, Ludeke, Haubrich, Gondan, \& Zettler, submitted; Liu, Ludeke, \& Zettler, 2017]. In one manuscript, we only included singles (whereas only participants in an intimate relationship are included here). In the other manuscript, we addressed a topic completely unrelated to the question of assumed similarity (namely, personality correlates of facets of authoritarianism).

ii There were no significant differences in the self-report HEXACO traits between participants included in this study (i.e., these being in intimate relationships) and participants excluded from this study (i.e., these not being in intimate relationships).

${ }_{\text {iii }}$ Results for all analyses based on Pearson correlations are presented in the supplementary material.

${ }^{\text {iv }}$ We also repeated these analyses for the other four HEXACO traits, and no significant interaction occurred.

${ }^{\mathrm{V}}$ We also repeated these analyses for the other four HEXACO traits, and there was no significant difference between China and Denmark. 